\title{
APLICAÇÃO DAS TRANSFORMADAS WAVELETS NA ANÁLISE DA QUALIDADE DA ENERGIA ELÉTRICA EM PROCESSOS INDUSTRIAIS
}

\author{
Jaime André Back ${ }^{1}$, Leonel Tedesco ${ }^{1,2, *}$, Rolf Fredi Molz ${ }^{1,2}$ \\ 1 Programa de Pós-graduação em Sistemas e Processos Industriais, Universidade de Santa Cruz do Sul (UNISC), 96815-900, Santa \\ Cruz do Sul-Brasil \\ 2 Departamento de Informática, Universidade de Santa Cruz do Sul (UNISC), 96815-900, Santa Cruz do Sul - Brasil
}

*E-mail: leoneltedesco@unisc.br

Recebido em: 07/09/2014 Aceito em: 07/10/2015

\section{RESUMO}

Este artigo apresenta o desenvolvimento de um sistema embarcado de baixo custo para a monitoração da qualidade da energia elétrica em ambientes industriais. Como o objetivo principal é a coleta de parâmetros elétricos que representam a qualidade da energia, os dispositivos de aquisição e rotinas de análise destes indicadores foram implementados através da utilização de técnicas de processamento digital de sinais (DSP). Por fim, para a detecção de perturbações elétricas, foi utilizada a Análise Multiresolução (AMR) a partir da aplicação das Transformadas Wavelets Discretas (TWD). Como resultado, obteve-se um sistema embarcado (hardware e software) para monitoração e análise da energia elétrica nos processos industriais A partir da análise dos dados coletados foi possível observar que a integração de diferentes tecnologias, associadas a metodologias e técnicas de captura e análise de sinais. Este trabalho resultou em ferramentas capazes de auxiliar na monitoração e no gerenciamento das demandas energéticas no setor industrial.

Palavras-chave: Qualidade da Energia Elétrica. Processamento Digital de Sinais. Transformadas Wavelets Discretas.

\section{Introdução}

O setor industrial brasileiro apresenta como uma de suas características a considerável demanda de energia elétrica. Em contrapartida a sua disponibilidade depende, principalmente dos níveis dos reservatórios que são utilizados por hidrelétricas na produção necessário ao consumo no país. Esta claro que a energia elétrica fornece a população uma série de benefícios [12], como na climatização de ambientes, residenciais e comerciais, principalmente, nos meses de verão, em que as temperaturas atingem os níveis elevados.

Pode-se dizer, então, que a qualidade da Energia Elétrica (QEE) é uma preocupação comum às empresas de energia elétrica. Concessionarias estatais e cooperativas de eletrificação, $\mathrm{e}$, aos consumidores de um modo em geral [12, 18]. Esse interesse pela QEE deve-se, principalmente, à evolução tecnológica de equipamentos eletroeletrônicos que são amplamente utilizados nos diversos segmentos da indústria, como a aplicação da eletrônica de potência, a automação de processos e a conversão de energia CA/CC.

No Brasil, a QEE afeta diretamente o setor industrial que consome, aproximadamente, $40 \%$ da energia elétrica gerada no país, conforme os dados da Confederação Nacional da
Indústria (CNI). Segundo [12, 14], a QEE, nos últimos anos, tornou-se o alvo de muitas pesquisas, realizadas tanto por universidades, pelo setor industrial, quanto pelas próprias concessionárias de energia elétrica. Ressalta que os fatores que influenciam na QEE podem ser originados tanto nas concessionárias como nos sistemas consumidores [14]. Os distúrbios, ou perturbações assim chamados, podem ser gerados, seja por fenômenos naturais, por operações da concessionária chaveamentos devido a operações com banco de capacitores e manutenções, seja pelos próprios consumidores, como excesso de cargas, acionamento de disjuntores, sistemas motrizes, baixo fator de potência entre outros.

Nesse contexto, torna-se importante que as indústrias tenham conhecimento das demandas e da qualidade da energia elétrica consumida diariamente, evitando tanto o desperdício quanto a diminuição da qualidade. Desse modo a QEE pode chegar ao consumidor com um excelente padrão de qualidade. Entretanto desde o mau gerenciamento a, problemas decorrentes da própria infraestrutura da empresa, podem implicar na diminuição da qualidade da energia que é consumida no processo industrial. 
Por isso objetivo do trabalho é apresentar o desenvolvimento de um sistema para a monitoração e o gerenciamento da energia elétrica em sistemas industriais que, possibilite o registro e a análise da energia elétrica consumida (EE), bem como os seus padrões de qualidade (QEE). Assim servindo, então, de ferramenta no auxílio à tomada de decisão a um melhor aproveitamento das demandas energéticas contratadas, principalmente em horário de ponta.

Para tanto trabalho está organizado em sete seções. A seção inicial compreende um apanhado de considerações sobre a qualidade da energia elétrica. Já a seção 3, detém-se o modelo matemático da Transformada de Wavelet Discreta. Na seção 4 é apresentada a Análise Multirresolução enquanto na seção 5 concentra-se os procedimentos metodológicos necessários à elaboração da pesquisa em questão. Por fim, a seção 6 fornece os resultados obtidos. A partir da discussão desses elementos, na seção 7 , apresenta-se a conclusão sobre o trabalho realizado e as direções para trabalhos futuros.

\section{Qualidade da Energia Elétrica}

O Brasil sofre constantemente com o risco de apagões. É principalmente, nos meses de alta temperatura e pouca chuva que o nível dos reservatórios das hidrelétricas é afetado e, consequentemente eleva o consumo de energia elétrica. $\mathrm{O}$ que se pode observar, como no gráfico a seguir, são os caminhos opostos que estão sendo traçados entre o consumo e o volume de água que é armazenada nos reservatórios. Percebe-se, então, que, enquanto o consumo aumenta, os níveis estão cada vez mais baixos

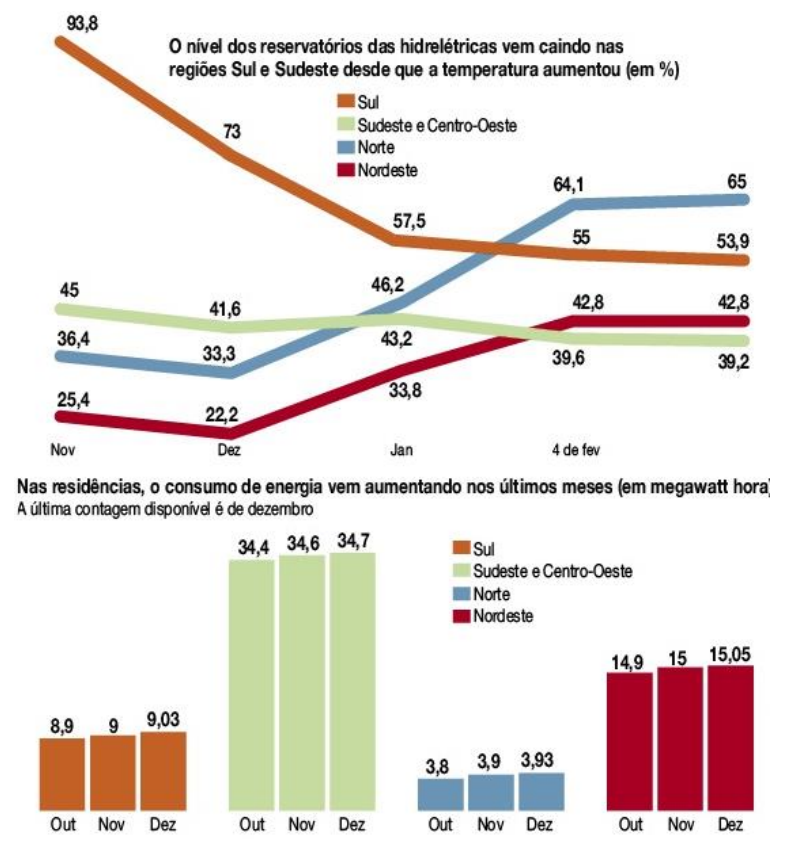

Figura 1 - Relação entre os níveis dos reservatórios e consumo de energia [16].
Dessa forma, a gestão de energia elétrica é um problema que atinge não apenas a sociedade em geral como também os diversos segmentos necessários à atividade humana. Uma vez que, tal fato influencia, além de despesas com a sua geração e manutenção, até o impacto ambiental de uma região, como em casos de criação de barragens, usinas hidrelétricas ou a carvão, e em colocação de linhas de distribuição.

No que diz respeito a termo, qualidade de energia elétrica, destaca-se que é utilizado para expressar as mais variadas características do que é entregue pelas concessionárias aos consumidores [3]. Essa medida inclui características de continuidade de suprimento e de conformidade com certos parâmetros considerados desejáveis à operação segura, tanto do sistema supridor como das cargas elétricas.

Em termos gerais, a QEE possui três níveis de qualidade, a saber: qualidade do atendimento, qualidade do serviço e qualidade do produto. A qualidade no atendimento refere-se às cobranças indevidas, taxas, tempo de atendimentoe entre outros. A qualidade no serviço está relacionada à operação e à manutenção do sistema elétrico que é proporcionado a clientes, o mínimo de interrupção aceitável. Já a qualidade no produto possui um foco técnico, que diz respeito à conformidade do produto, ou seja, a disponibilidade de energia elétrica com tensões senoidais equilibradas e com amplitude constante $[4,6,8]$.

\subsection{Monitoração da Qualidade da Energia Elétrica}

Conforme [3], é necessário que os técnicos ou especialistas façam uma pesquisa, estudo, para diagnosticar as causas dos problemas relativos à qualidade da energia. Então, como se trata de diagnosticar um problema de compatibilidade eletromagnética ou, ou até mesmo a busca por indicadores de parâmetros elétricos fora de padrões, esta pesquisa pode envolver questões que estão além de um simples problema tecnológico. Em virtude disso, uma abordagem recomendável poderia observar:

- Em primeiro lugar,conhecer os problemas que se poderá enfrentar;

- Estudar as condições locais onde o problema se manifesta;

- Se possível, medir e registrar as grandezas contendo os sintomas do problema;

- Analisar os dados e confrontar os resultados obtidos com estudos ou simulações;

- Finalmente, diagnosticar o problema, sua possível causa, propondo soluções.

Portanto, a QEE refere-se a uma ampla variedade de fenômenos eletromagnéticos conduzidos que caracterizam a tensão e a corrente num dado tempo e local do sistema elétrico, como mostra a Figura 2. Assim, conforme os autores [3, 6, 18], a qualidade da energia em uma determinada região do sistema 
elétrico é adversamente afetada por uma ampla variedade de distúrbios, tais como:

- Transitórios (impulsivos e oscilatórios);

- Variações de curta duração (interrupções transitórias, afundamentos de tensão e saltos de tensão);

- Variações de longa duração (interrupções sustentadas, subtensões e sobretensões);

- Distorção de forma de onda (corte de tensão, harmônicos, ruído, etc.);

- Flutuações de tensão e variações de frequência.

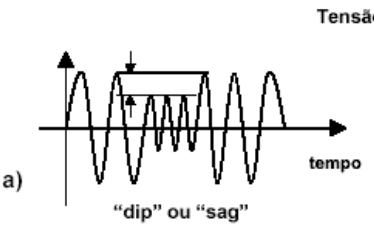

Tensăo de rede

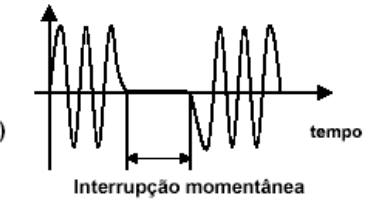

b)

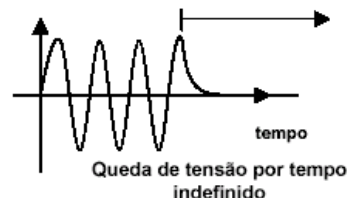

d)

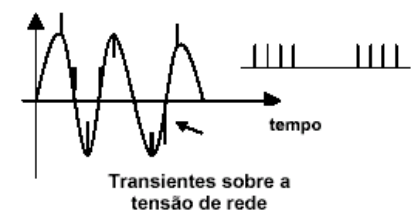

Figura 2 - Principais distúrbios associados à QEE [6].

2.2 Principais métodos utilizados na identificação de parâmetros de QEE nos sistemas industriais

Para Ferreira [6], a identificação e classificação de distúrbios elétricos representa uma importante etapa em um processo de monitoramento da QEE, uma vez que pode contribuir diretamente na identificação das causas dos distúrbios.

Conforme os trabalhos [5, 8], para a identificação de um distúrbio elétrico são necessárias funções ou algoritmos que permitam a extração de parâmetros que caracterizem estes eventos. Assim, são utilizados os seguintes métodos na identificação de distúrbios elétricos:

- Método baseado no cálculo do Valor RMS;

- Método baseado na Norma Euclidiana Instantânea;

- Método baseado na aplicação da Transformada Discreta de Fourier;

- Método baseado na aplicação da Transformada Wavelet.

A Transformada Wavelet é utilizada quando se necessita localizar eventos isolados no domínio do tempo [13], visto que o método clássico de análise de espectro de frequência usando a Transformada de Fourier é adequado para sinais periódicos em regime permanente. A Transformada Wavelet possui suas variantes contínua (TWC) e discreta (TWD).

\section{A Transformada Wavelet Discreta}

Segundo Filho [7], a discretização dos parâmetros $a$ e $b$ de uma TWC origina a Transformada Wavelet Discreta (TWD). A discretização do parâmetro de translação depende da escala da wavelet mãe. Assim, para as altas frequências, as wavelet mães são transladadas por passos pequenos, enquanto que para as frequências baixas, a translação é feita com passos maiores [17].

Matematicamente, a definição da TWD de uma função de expansão para qualquer sinal em $L^{2} R$ pode ser representada pela equação 1 , conforme [2]

$$
f(t)=\sum_{j-k} a_{j, k} 2^{j / 2} \psi\left(2^{j} t-k\right)
$$

Ou simplificando, como na equação 2 [2].

$$
f(t)=\sum_{j-k} a_{j, k} \psi_{j, k}(t)
$$

Uma forma mais específica é apresentada na equação 3 [2], que indica como os coeficientes $a_{j, k}$ são calculados, pode ser escrita usando produto interno.

$$
f(t)=\sum_{j-k}\left\langle\psi_{j, k}(t), f(t)\right\rangle \psi_{j, k}(t)
$$

Onde $\psi_{j, k}(t)$ forma uma base ortonormal para o espaço de sinais de interesse. Por fim, a TWD também pode ser escrita conforme equação 4 [2], onde $m$ é a escala $n$ a translação.

$$
T W D(m, n)=\left|a_{0}{ }^{m}\right|^{\frac{-1}{2}} \sum_{n} f(n) \psi\left(\frac{x-n b_{0} a_{0}{ }^{m}}{a_{0}{ }^{m}}\right)
$$

\section{Análise Multirresolução}

O efeito da mudança de escala de um sinal pode ser melhor interpretado usando-se o conceito de resolução. Tal efeito, consegue-se através do uso de filtros, [6]. processo de filtragem utilizado para a TWD apresenta uma forma de realização da técnica de Análise Multirresolução (AMR) proposta por Mallat, tendo como resultado a combinação de uma função de escala $f_{(t)}$ e de uma função wavelet $\psi_{(t)}$. 


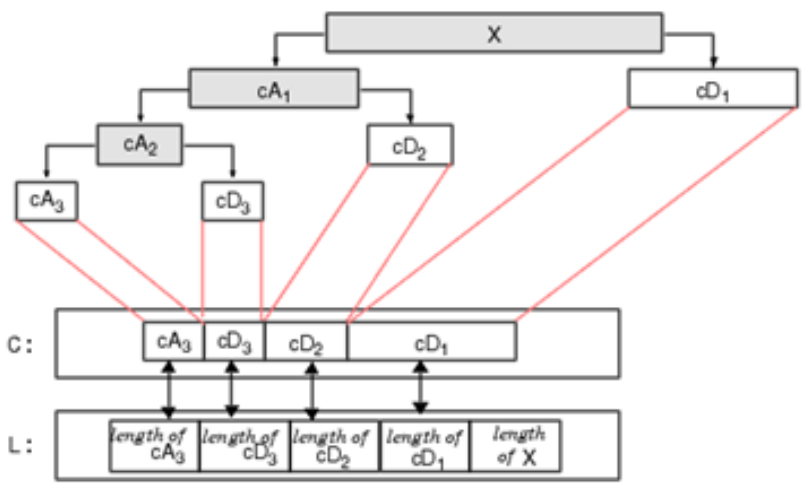

Figura 3 - Algoritmo piramidal de Mallat.

Para Filho [7] e Garcia et al. [8], é possível separar componentes de frequência dos sinais utilizando os filtros de decomposição wavelet (Figura 3). Este processo baseia-se na filtragem de um sinal através de filtros passa-alta (detalhes) e passa-baixa (aproximações), fornecendo versões do sinal original relativas aos coeficientes de funções wavelets e funções escala, ou aproximações e detalhes [15], respectivamente, como apresentado pela Figura 4.

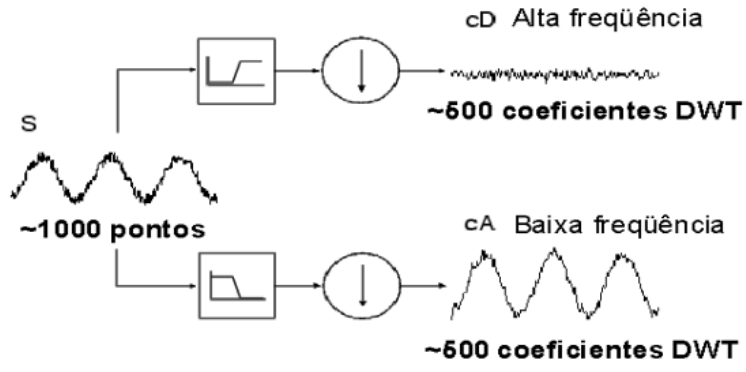

Figura 4 - TWD de um estágio para um sinal senoidal com ruído [15].

A TWD multirresolução seguiu a estrutura apresentada na Figura 2. Já a utilização da wavelet mãe de Haar corresponde à multiplicação matricial, conforme equação 5 [9].

$$
\begin{gathered}
S_{0} \\
{\left[\begin{array}{cc}
k_{1} & K_{2} \\
k_{1} & K_{2} \\
\cdots & \cdots \\
k_{n-1} & K_{n}
\end{array}\right] \times\left[\begin{array}{cc}
C_{0} & C_{0} \\
C_{1} & -C_{1}
\end{array}\right]=\left[\begin{array}{ll}
a_{m} & d_{m} \\
a_{m} & d_{m} \\
\cdots & \ldots . \\
a_{m} & d_{m}
\end{array}\right]} \\
\text { Onde } c_{0}=\frac{\sqrt{2}}{2} \text { e } c_{1}=\frac{\sqrt{2}}{2} \text { (coeficientes wavelet de Haar). }
\end{gathered}
$$

\section{Metodologia}

Para atingir os objetivos de desenvolver um equipamento, e uma metodologia, voltados ao monitoramento da qualidade da energia elétrica, este projeto foi dividido em três fases principais, conforme descrito na sequência.

$\mathrm{Na}$ primeira etapa, foi desenvolvido o sistema de aquisição e análise de dados provenientes do sistema de fornecimento de energia elétrica. A etapa compreende o projeto e o dimensionamento das placas eletrônicas de condicionamento de sinais, das placas de aquisição e discretização dos dados e a programação dos sistemas embarcados.

A segunda etapa correspondeu à instalação e calibração do sistema de aquisição no ambiente industrial. Este sistema permaneceu em funcionamento durante cinco semanas, nas quais coletou parâmetros de tensão e corrente de 3 transformadores, com capacidade de até 1000 Ampères cada.

$\mathrm{Na}$ terceira e última etapa, os dados coletados pelo sistema foram analisados, podendo, assim, ser desenvolvido o modelo de consumo e, também, foi implementada uma aplicação computacional para detecção e classificação dos distúrbios elétricos através da aplicação das Transformadas Wavelets.

\subsection{Implementação do sistema de aquisição}

Como proposto por Back e Tedesco [1], a aquisição dos parâmetros elétricos pode ser realizada com a utilização de transformadores de corrente (TC) e transformadores de potencial (TP) conectados à rede elétrica (correspondendo à etapa de entrada de sinal). Este sinal é condicionado para atingir faixas de valor específicos e dentro dos limites do conversor analógico digital (ADC), utilizado no processamento destes sinais. Como saída, tem-se um sinal digitalizado, ou valor discretizado, podendo, assim, ser manipulado computacionalmente. 

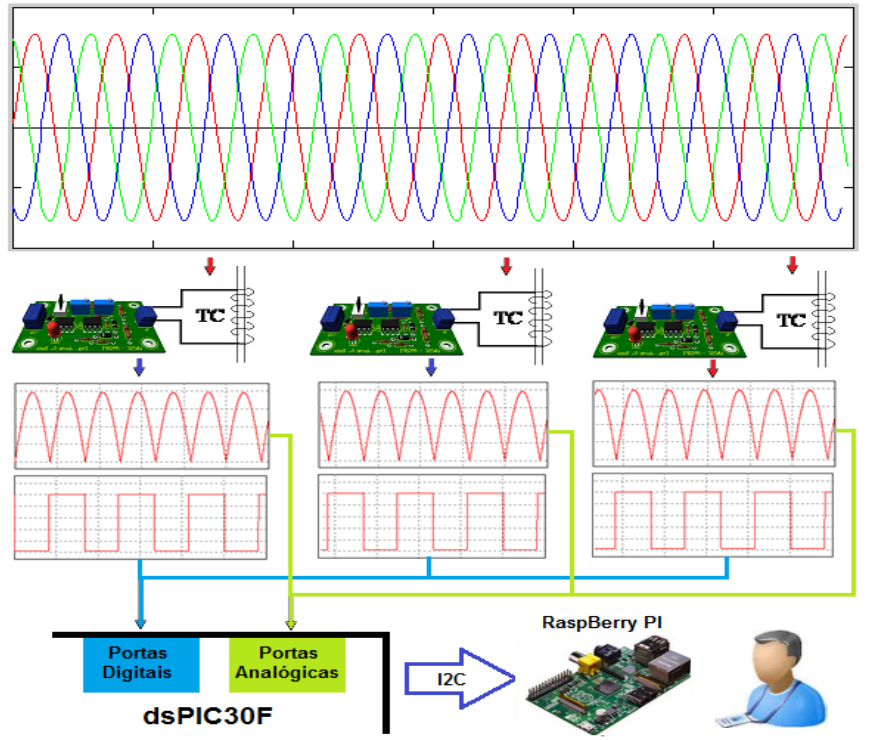

Figura 5 - Fluxo de sinais no cenário estudado

A Figura 5 apresenta o cenário de estudos e descreve mais detalhadamente a segunda e a terceira etapas, em que é possível observar os sinais senoidais de entrada (SINAL DO BARRAMENTO), que são capturados pelos sensores (LEITURA) e, posteriormente, condicionados de forma a se ter dois sinais distintos: um sinal retificado e um sinal digital (CONDICIONAMENTO). Ambos sinais são lidos pelo circuito DSP (dsPIC30F), discretizados e enviados ao sistema embarcado (Raspberry PI) para serem visualizados pelo usuário (DISCRETIZAÇÃO).

A última etapa do desenvolvimento do sistema corresponde à integração de todos os dispositivos já desenvolvidos neste projeto. Logo, para iniciar a monitoração de um sistema de produção trifásico, necessita-se de:

- Plataforma Embarcado RaspBerry PI;

- Placa microcontrolada para conversão AD;

- Condicionadores para os sinais dos TCs;

- Condicionadores para os sinais dos TPs;

- Conversor para comunicação I2C entre os dispositivos;

- Fonte de alimentação.

\subsection{Algoritmo AMR embarcado}

Um algoritmo para análise de perturbações utilizando TWD foi desenvolvimento em linguagem $\mathrm{C}$, dispensando a necessidade da utilização de software proprietário, como o Matlab, além da possibilidade de se portar o algoritmo a qualquer tipo de plataforma embarcada.

A Figura 6, apresenta o diagrama de blocos para análise de um sinal utilizando AMR. Destaca-se que é importante notar os tempos de discretização, e a aplicação dos filtros passa-baixa e passa-alta no sinal amostrado (sinal proveniente do sistema de aquisição).

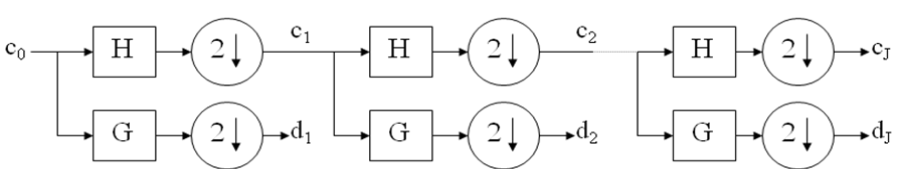

Figura 6 - Diagrama de blocos para análise AMR do sinal.

Uma forma prática de realizar a AMR é através do algoritmo piramidal de Mallat (Figura 3), que consiste em dividir o sinal original em outros dois sinais: um deles corresponde ao sinal original suavizado e o outro à uma ampliação das oscilações ou "ruídos" do sinal analisado [13]. Para a implementação do algoritmo do sistema embarcado, o fluxograma, apresentado na Figura 7, teve que ser executado. Neste fluxograma, a função de Haar é aplicada ao sinal $S$ e dois subsinais são gerados. O sinal $H(s)$ representa os coeficientes wavelet resultantes da aplicação da Transformada Wavelet Haar.

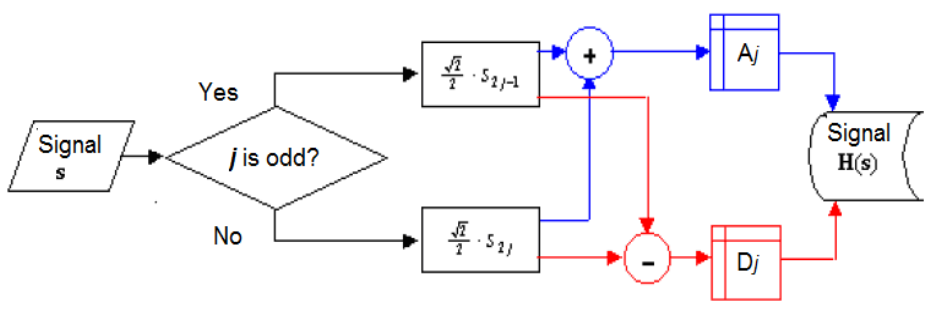

Figura 7 - Análise de elevação de tensão por TWD.

Para validação do algoritmo desenvolvido para o sistema embarcado, o sinal original foi analisado utilizando o software Matlab 7.0, conforme apresentado na Figura 8. É possível observar a similaridade entre os gráficos dos sinais de decomposição resultantes da aplicação dos dois algoritmos, algoritmo do Matlab e o algoritmo do sistema embarcado. Em ambos os casos, as perturbações na curva de consumo foram apresentadas com localização e intensidade iguais. 


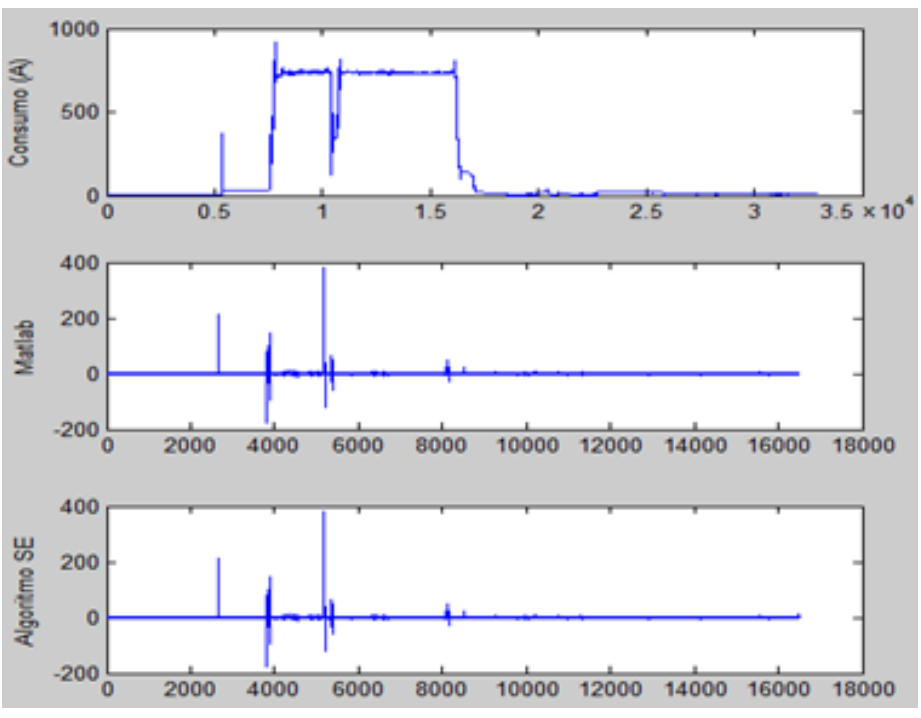

Figura 8-Análise AMR dos dados de consumo.

\section{Resultados e discussões}

Para validação do projeto desenvolvido, foi executado, como estudo de caso, o monitoramento da qualidade da energia elétrica e da demanda energética consumida em um processo de beneficiamento de tabaco. Escolheu-se este cenário de estudo pelo alto consumo de energia em seus processos, além da grande quantidade de elementos de potência instalados, como motores de indução trifásicos, caldeiras, reatores e, entre outros.

Dessa forma, o processo em que o sistema de monitoramento foi instalado corresponde à linha de beneficiamento de fumo, que é caracterizada pelas seguintes cargas elétricas, em outras palavras, cargas de maior relevância: 280 motores de indução trifásicos; 250 motores são acionados por partida direta; 32 motores são controlados por inversores de frequência.

\subsection{Análise de consumo}

Esta etapa corresponde à verificação do comportamento do consumo de energia elétrica pelo sistema e sua eficiência. Os dados a serem analisados correspondem ao valor de corrente consumida em cada fase dos transformadores monitorados pelo sistema de aquisição dos mesmos. O sistema implementado monitorou o processo industrial por, aproximadamente, um mês. Ressalta-se que neste período, a empresa operou em turno único, com horário de funcionamento das 06:20 horas até 13:30 horas.

Os dados coletados permitiram a verificação da QEE, bem como, traçar o modelo de consumo do processo. Por exemplo, a Figura 9 demonstra o comportamento da demanda energética monitorada e registrada durante um dia de produção.
$\mathrm{Na}$ imagem, é possível observar a similaridade entre as três curvas, que representam a demanda de três transformadores instalados na subestação.

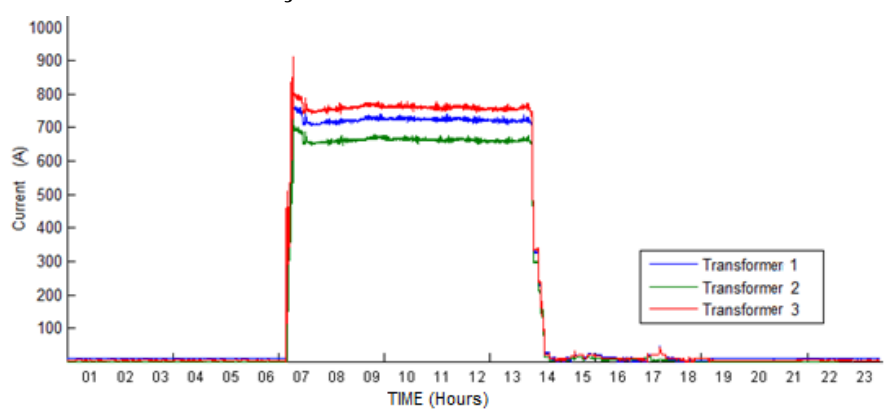

Figura 9 - Gráfico com as correntes IR de três transformadores

\subsection{Análise de QEE}

Os parâmetros de QEE na corrente elétrica também afetam os padrões de consumo, devendo ser considerados em todos os processos de análise e monitoração. Neste trabalho, estas perturbações foram analisadas a partir dos valores de consumo médio, ou seja, os valores de corrente eficaz $\left(\mathrm{I}_{\mathrm{RMS}}\right)$ a cada uma das fases dos transformadores.

Estas perturbações correspondem a eventos abruptos, que ocorrem instantaneamente, modificando o comportamento e a curva de consumo do sistema. Pode-se citar, por exemplo, o acionamento de grandes cargas, como os motores de indução por partida direta, que geram picos de corrente que podem atingir valores até dez vezes a corrente nominal. Da mesma forma, o desligamento de dispositivos também causa diminuição repentina da corrente, ou afundamentos.

A Figura 10.a apresenta a curva de consumo diário do processo industrial em que é possível observar um afundamento na corrente, que foi causado pelo desligamento repentino dos equipamentos e máquinas da linha de produção devido a uma sobre-tensão, quadro em detalhe, no qual a tensão nominal da rede chegou a 245 Volts. Com base nesta curva de consumo, é aplicada a TWD de modo a localizar os distúrbios de corrente. Como resultado, tem-se o gráfico de espectro wavelet (Figura 10.b), correspondendo ao ruído presente na curva de consumo após a análise utilizando wavelet mãe de Haar. Para esta análise, os pontos mais importantes do gráfico são os saltos de maior amplitude, que indicam os momentos em que ocorreram as mudanças abruptas na curva de consumo.

Por fim, ainda com base na Figura 10.a, nota-se o tempo preciso para a reestabilização do processo, ou seja, o tempo necessário para que o comportamento da curva de consumo se ajuste ao seu modelo característico (próxima de 800 A). Neste período, que teve duração de aproximadamente 40 minutos, os 
funcionários foram obrigados a parar com a realização de suas atividades. Por consequência, tabaco não pôde ser beneficiado.
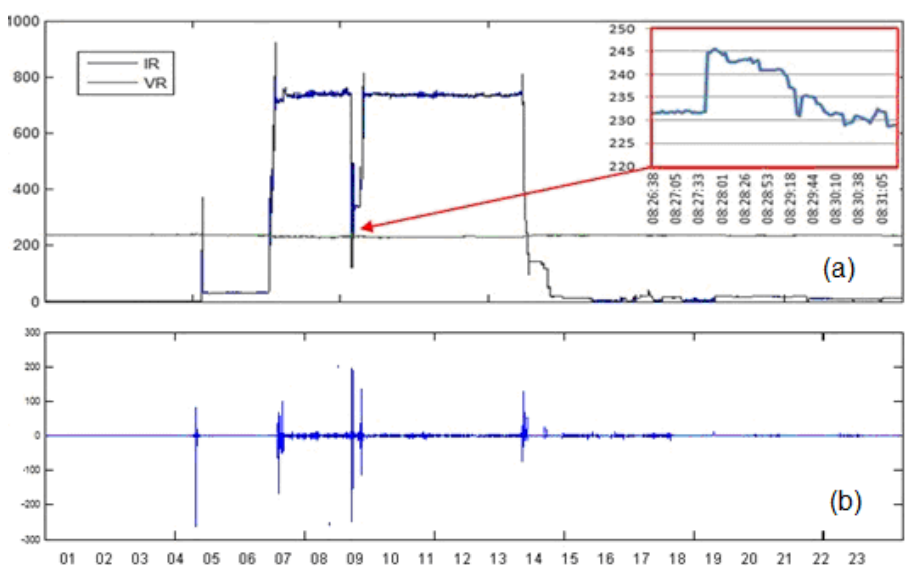

Figura 10 - (a) Análise de perturbação. (b) Aplicação da TWD Haar

Já a análise de perturbações de QEE dos dados de tensão senoidal coletados pelo sistema embarcado (para uma única fase) pode ser visualizada na Figura 11, na qual é possível observar a mudança no nível de espectro wavelet no momento em que a amplitude da senoide se eleva repentinamente (elevação de tensão de curta duração). Este impulso, na resposta do espectro, corresponde aos coeficientes de detalhe quando inserido uma perturbação no sinal original e são facilmente detectados pela função wavelet, devido sua característica de análise no domínio do tempo.
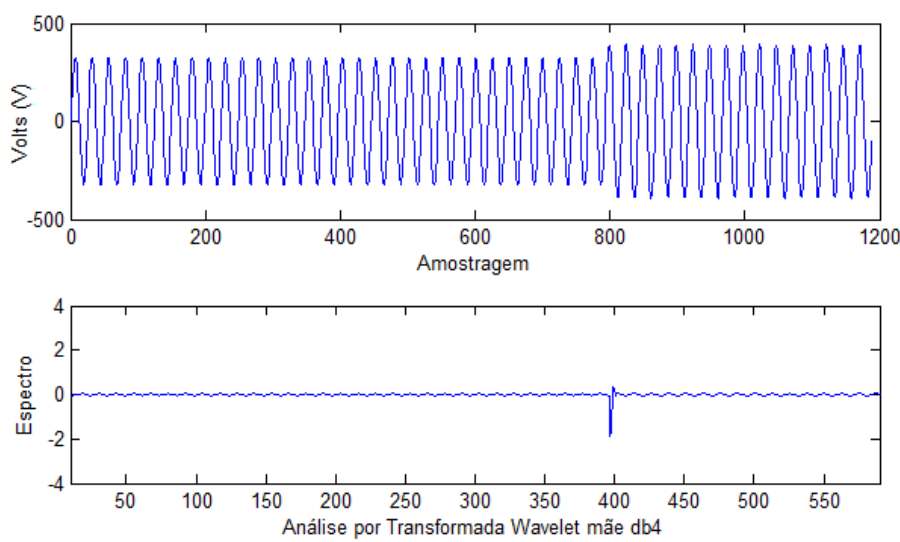

Figura 11 - Análise de elevação de tensão por TWD

\section{Conclusões}

Neste trabalho apresentaram-se os resultados alcançados com o desenvolvimento de um sistema embarcado voltado ao gerenciamento da energia elétrica em processos industriais. A partir da análise dos dados coletados foi possível observar que a integração de diferentes tecnologias, associadas a metodologias e técnicas de captura e análise de sinais, resultam em ferramentas capazes de auxiliar na monitoração e no gerenciamento das demandas energéticas no setor industrial.

A análise da qualidade e da demanda consumida (demanda total, por área, setor, horário) pode fornecer o modelo parcial, porém capaz de ilustrar o comportamento do consumo elétrico do processo industrial. $\mathrm{O}$ algoritmo desenvolvido para a análise de demanda possibilita, além da verificação da demanda consumida, a criação de perfis de consumo para cada dia ou semana, de forma a estimar picos de consumo, evitando, assim, ultrapassar a demanda contratada.

No entanto, para trabalhos futuros, deseja-se aprimorar o armazenamento dos dados a partir de um banco de dados que torne essas informações acessíveis aos sistemas de gestão. Também deseja-se substituir os sistemas de leitura de dados por sistemas mais rápidos, já que a utilização de microcontroladores apresentou limitações na transferência dos dados. Por fim, destaca-se que será necessária a implementação de novas rotinas de análise AMR, utilizando, por exemplo, as wavelets mãe Daubechies (db4), em virtude de que a Haar não obteve os resultados satisfatórios à análise de QEE para sinais senoidais.

\section{APPLICATION OF WAVELET TRANSFORM IN ANALYSIS OF POWER QUALITY IN INDUSTRIAL PROCESSES}

ABSTRACT: This article presents the development of a low cost embedded system for monitoring the quality electrical power in industrial environments. As the primary purpose is the collection of parameters that represent the electric power quality devices, acquisition and analysis routines of these indicators have been implemented through the use of techniques of digital signal processing (DSP). Finally, for the detection of electrical disturbances, we used the Multiresolution Analysis (AMR) from the application of the Discrete Wavelet Transform (TWD). As a result, we obtained an embedded system (hardware and software) for monitoring and analysis of electric energy in industrial processes. Based on collected data, it was possible to observe the effect of different technologies integration, associated to methodologies and techniques for signal capturing and analysis. This work resulted in tools, which will be used to help in the process of monitoring and management of industrial sector energetic demands.

Keywords: Electric Power Quality. Digital Signal Processing. Discrete Wavelet Transform. 


\section{Referências}

[1] BACK, J. A.; TEDESCO, L. P. Proposta de um Sistema para Monitoração e Analise de Energia Elétrica em Ambientes Industriais. In: XXXII ENEGEP, Bento Gonçalves - RS, Brasil. Anais... Bento Gonçalves, 2012.

[2] BURRUS, C. S.; GOPINATH, R. A.; GUO, H. Introduction to wavelets and wavelet transforms. New Jersey, Prentice Hall, 1998.

[3] DECKMANN, S. M.; POMILIO, J. A. Avaliação da Qualidade da Energia Elétrica. 2010, Material Didático - PPG em Engenharia Elétrica - Unicamp, <http://www.dsce.fee.unicamp.br/ antenor/pdffiles/qualidade/a1. pdf>. 2011.

[4] DUGAN, R. C.; MCGRANAGHAN, M. F.; SANTOSO, S.; BEATY, H. W. Electrical Power Systems Quality. 2nd ed., New York: McGraw Hill, 2003.

[5] DUQUE, C. A., RIBEIRO, M. V., RAMOS, F. R., et al. Power Quality Event Detection Based on the Divide and Conquer Principle and Innovation Concept. IEEE Transactions on Power Delivery, v. 20, n. 4, pp. 2361 - 2369, 2005.

[6] FERREIRA, J. C. Utilização da Transformada de Wavelet para Detectar Variações Anormais de Frequência em Sistemas de Geração Distribuída. Dissertação de Mestrado - Programa de Pós-Graduação em Engenharia Elétrica, Universidade Federal de Uberlândia, Uberlândia, 2009.

[7] FILHO, O. D. Utilização da transformada wavelet para caracterização de distúrbios na qualidade da energia elétrica. Dissertação de Mestrado - Escola de Engenharia da USP São Carlos, 2003.

[8] GARCIA, V. V.; KAGAN, E. L.; AMASIFEN, J. C. C.; SUEMATSU, A. K.; HAYASHI, R.; CHAVES, L. B. Sistema de Consultoria de Qualidade de Energia Elétrica. In: VIII Conferência Brasileira sobre Qualidade da Energia Elétrica, Blumenau - SC, Brasil. Anais... Blumenau, 2009.

[9] GOMES, J., VELHO, L., GOLDENSTEIN, S. Wavelets: Teoria, Software e Aplicações. In: $21^{\circ}$ Colóquio Brasileiro de Matemática, Rio de Janeiro, IMPA, p.216. 1997.

[10] HUA, L.; BUAGUN, Z.; HONG, Z., Recognition and Classification of Power Quality Event in Power System Using Wavelet Transform. Proceedings of 27th Chinese Control Conference, pp. 43-46, 2008.

[11] JUNIOR, O. H. A. Desenvolvimento de uma Metodologia para Identificar e Quantificar Distúrbios da Qualidade da Energia Elétrica. 2009. 188 p. Dissertação de Mestrado (PPGEEUFRGS), Porto Alegre, 2009.
[12] LEBORGNE, R. Uma Contribuição à Caracterização da Sensibilidade de Processos Industriais Frente a Afundamentos de Tensão. 2003. 163 p. Dissertação de Mestrado (CPG-E), 2003.

[13] MALLAT, S. G. A theory for multiresolution signal decomposition: The wavelet representation. IEEE Transactions on Pattern Analysis and Machine Intelligence, v. 11, n. 7, p. 674$693,1989$.

[14] MELO, M. O. B. C. Avaliação do impacto da qualidade de energia elétrica na produção industrial: proposta de metodologia. In: Revista Produto \& Produção, vol. 9, no 3, pp. 15- 25, 2008.

[15] MISITI, M., MISITI, Y., OPPENHEIM, G., POGGI, J. Wavelet Toolbox For Use with MATLAB ${ }^{\circledR}$. User's Guide, Revised for Version 4.10 (Release 2012b), The MathWorks, Inc., 2012.

[16] ONS. Operador Nacional do Sistema Elétrico. 2006. Disponível em: <http://www.ons.org.br/home/>. Acesso em: 2014.

[17] SANTOSO, S.; GRADY, W. M.; POWERS, E. J.; et al. Characterization of distribution power quality events with Fourier and wavelet transforms. IEEE Transactions on Power Delivery, v. 15, n. 1, pp. 247-254, 2000.

[18] SOLÓRZANO, K. M. L. Uma Contribuição ao Estudo de Sobretensões em Sistemas Elétricos de Pequeno Porte Contendo Cargas Não - Lineares. Dissertação de Mestrado, Universidade Federal de Itajubá, 2004. 